\title{
DIZZYNET 2018: visions and perspectives of future vestibular research
}

\author{
Andreas Zwergal ${ }^{1,2} \cdot$ Thomas Brandt $^{2,3} \cdot$ Hans Straka $^{2,4} \cdot$ Dominik Straumann ${ }^{5} \cdot$ Marianne Dieterich $^{1,2}$
}

Received: 27 August 2018 / Accepted: 27 August 2018 / Published online: 6 September 2018

c) Springer-Verlag GmbH Germany, part of Springer Nature 2018

Keywords Networking $\cdot$ Vertigo $\cdot$ Dizziness $\cdot$ Balance disorders $\cdot$ Vestibular

Recent advances in vertigo and balance research have surpassed the classical investigative approaches to vestibular function and dysfunction. In addition to well-defined lab conditions, novel psychophysical, neurophysiological, functional imaging, and modeling techniques allow researchers to study the vestibular system of animals and humans in different natural environmental contexts. Computational sciences now offer unexpected possibilities for analyzing and modeling big data sets, which, however, demand supportive structures to promote the necessary collaboration and data collection. The DIZZYNET community has responded with imaginative ideas to help the various European expert centers face this challenge together.

In the current supplement, Eva Grill and colleagues describe first steps towards a web-based data storage system in clinical vestibular research. The long-term goal is to acquire and store large data sets for analyzing diseasespecific patterns based on computational algorithms. Data banking of parameters derived from apparative testing such as video-oculography will complement this process.

This manuscript is part of a supplement sponsored by the German Federal Ministry of Education and Research within the funding initiative for integrated research and treatment centers.

Andreas Zwergal

andreas.zwergal@med.uni-muenchen.de

1 Department of Neurology, Ludwig-Maximilians-University, Marchioninistrasse 15, 81377 Munich, Germany

2 German Center for Vertigo and Balance Disorders, DSGZ, Ludwig-Maximilians-University, Munich, Germany

3 Clinical Neurosciences, Ludwig-Maximilians-University, Munich, Germany

4 Division of Neurobiology, Department of Biology, Ludwig-Maximilians-University, Munich, Germany

5 Department of Neurology, University Hospital Zurich, Zurich, Switzerland
This year's DIZZYNET issue of the Journal of Neurology presents contributions of members from all fields of basic, translational, and clinical vestibular research. Several articles focus on the basic function and clinical application of the vestibulo-ocular reflex. Hans Straka and colleagues, for example, report their findings on the pharmacology of plane-specific vestibular inhibitory inputs to extraocular motoneurons. Other members address the advances and novel developments in video-oculography for clinical testing of the vestibulo-ocular reflex, stressing in particular its potential methodological problems and applications in the acute vestibular syndrome. Two interesting case reports illustrate the potential of contrast-enhanced inner ear imaging for differential diagnoses of vestibular disorders. Antonio Lopez-Escamez and colleagues summarize the genetic contributions to vestibular pathophysiology. Several articles focus on neuromodulatory approaches to the treatment of vestibular disorders; for example, galvanic vestibular stimulation or stimulation of the vagal nerve. Others discuss advanced strategies that apply virtual environments and somatosensory substitution to treat patients with postural imbalance due to vestibular disorders or stroke. A number of articles examine unknown aspects of higher vestibular function. Christophe Lopez and colleagues report that patients with dizziness experience a distorted representation of the body. Another paper reveals that a pronounced susceptibility to motion sickness is a common comorbidity of various vestibular disorders. Two conceptual papers by Thomas Brandt, Marianne Dieterich, and Stefan Glasauer shed light on the separate operations of the bilateral thalamo-cortical vestibular networks and model the neuronal networks for the perception of verticality. Finally, a position paper involving a multinational team of researchers emphasizes that the laws regulating driver's licenses for patients with vertigo and dizziness need to be conformed to a common standard recognized by all of Europe. 
The above-mentioned articles demonstrate the specific advantages of the international DIZZYNET as a collaborative platform for launching future vertigo and balance research, for it is the cooperation of researchers with diverse backgrounds that leads to productive scientific synergies.

Acknowledgements The DIZZYNET initiative was supported by the German Federal Ministry of Education and Research (BMBF) within the context of the foundation of the German Center for Vertigo and Balance Disorders (DSGZ) (grant number 01 EO 0901). The authors thank Judy Benson for critically reading and copy-editing the manuscript.

\section{Compliance with ethical standards}

Conflicts of interest The authors declare no competing financial interests. 